\title{
Heilerpersönlichkeit und Heilungsbereitschaft der Hilfesuchenden
}

\section{Horst H. Figge}

\section{Einleitung}

Bei Überlegungen zur Heilung von Krankheiten steht der Einfluß des Heilers und seiner Mittel meist sehr stark im Vordergrund der Aufmerksamkeit. Es kann geradezu in Vergessenheit geraten, daß es der lebende Organismus des Patienten selbst ist, der heilt. Außenstehende, ganz gleich welche Mittel sie auch einsetzen mögen, können nichts anderes tun, als den Selbstheilungsprozeß des Organismus zu fördern. Dieser Selbstheilungsprozeß setzt zweierlei voraus: 1. die objektive Heilungsfähigkeit des Patienten, 2. seine subjektive Heilungsbereitschaft. Beide müssen gleichzeitig gegeben sein, damit eine Heilung zustandekommt; beide sind aber auch unabhängig voneinander von außen förderbar.

Die chirurgische, apparative, medikamentöse Medizin richtet ihr Bemühen auf die Lenkung und Unterstützung der Heilungsfähigkeit des Patienten. Seine Heilungsbereitschaft setzt sie als gegeben voraus. Es liegt außerhalb der so verstandenen Medizin, daß ein Patient sich verordneten Maßnahmen nicht unterzieht, auf objektiv wirkende Medikamente nicht in der erwarteten Weise anspricht, nach einer gelungenen, komplikationsfreien Operation stirbt. Extremes Beispiel für die Betrachtung der Heilungsbereitschaft als Störfaktor ist der Doppelblindversuch, in dem es nicht mehr um einen zu heilenden Menschen geht, sondern letzlich um chemische Wirkungen auf chemische Prozesse.

Sozusagen am entgegengesetzten Pol steht der Heilzauber; er richtet sein Bemühen auf die Weckung und Stärkung der Heilungsbereitschaft (wenn seine Vertreter das auch anders sehen mögen). Er verläßt sich ganz auf die vorhandene Heilungsfähigkeit des Patienten. Ihr Fehlen stellt dann für ihn den Störfaktor dar, dem er nichts entgegenzusetzen hat. 
Traditionelle Heiler besitzen nur wenige Mittel, die Heilungsfähigkeit zu lenken und zu unterstützen; sie behandeln nicht die Krankheit, sondern den Kranken. Hier liegt auch der Grund für die oft kritisierte unscharfe Indikation und unzureichende Reproduzierbarkeit ihrer Methoden und Erfolge. Derartige Forderungen aufzustellen, geht gänzlich an der Sache vorbei, um die es eigentlich geht. Die Heilungsbereitschaft ist per se ein völlig subjektiver Faktor; sie bewirkt den tatsächlichen Einsatz der vorhandenen individuellen Heilungsfähigkeit im Rahmen der aktuellen subjektiv erlebten Gesamtsituation des Patienten. Die Extremform der Gesundheitsgefährdung und zerstörung aus mangelnder Heilungsbereitschaft, bei der die Heilungsfähigkeit überhaupt keine Rolle spielt, liegt im Suizidversuch vor. Andererseits ist die Extremform der Förderung der Heilungsfähigkeit, bei völliger Bedeutungslosigkeit von Heilungsbereitschaft, gegeben, wo der psychisch tote Körper oder gar nur Teile davon in 'gesunder' Existenz gehalten werden.

Wenn trotz aller inzwischen extremen Möglichkeiten unserer Medizin, die Heilungsfähigkeit des Menschen zu beeinflussen, Krankheit eben nicht verschwunden ist, wird das auch nur daran liegen, daß das andere Ursachenbündel, nämlich die Heilungsbereitschaft, bisher unzureichend berücksichtigt worden ist. Unzureichende Heilungsbereitschaft führt zum Nichteinsatz der vorhandenen Heilungsfähigkeit, der Abwehrkräfte gegen krankmachende Agentien oder sogar zum gezielten Aufsuchen von Situationen, in denen die Heilungsfähigkeit überfordert ist. Daß hierbei Bewußtheit, Einsicht, Wille, erlebte Absicht meist keine Rolle spielen, braucht kaum erwähnt zu werden.

\section{Überlegungen zur Heilungsbereitschaft}

Worum es sich bei der Heilungsbereitschaft handelt, läßt sich am einfachsten negativ über die Faktoren erklären, die sie herabsetzen. Ich möchte zunächst die verschiedenen Formen von Krankheitsgewinn nennen. Die Begriffe des primären und 
sekundären Krankheitsgewinns sind von Sigmund FREUD eingeführt worden; sie werden hier mit teilweise abweichender Bedeutung verwendet. (Zur Zusammenfassung des Freudschen Verständnisses siehe BALLY 1961: 171-173).

1. Primärer Krankheitsgewinn: Krankheit kann von anderen unangenehmeren Problemen ablenken; über sie kann man insbesondere inneren Konflikten ausweichen. Krankheit dient damit gegebenenfalls zur Stabilisierung der eigenen Persönlichkeit, wie die Psychoanalyse deutlich genug gezeigt hat. Krankheit kann auch äußerlich faszinieren, die Beschäftigung mit der eigenen Krankheit zum Ersatz für anderweitig fehlende Aufgaben werden.

2. Sekundärer Krankheitsgewinn: Die vom Kranken erwartete und übernommene Rolle enthält eine Reihe von unter Umständen sehr attraktiven Momenten, da Pflichten wegfallen, Rechte erworben werden. Die gewährten Rücksichten und Hilfen halten dann die Krankheit aufrecht, da sie ohne sie nicht mehr gewährt würden.

3. Tertiärer Krankheitsgewinn: Auch für die beteiligte Gruppe und ihre Mitglieder kann die Krankheit des einzelnen eine Fülle wünschenswerter Folgen haben. Sie schafft z.B. Aufgaben, erhöht das Selbstwertgefühl der Helfenden, stellt gegebenenfalls deren Existenzgrundlage dar. Sie lenkt von Gruppenproblemen ab und wirkt damit gruppenerhaltend und -stabilisierend; siehe Sündenbockrolle, der Kranke als Symptomträger der Gruppe. Über entsprechende Rückmeldung ergibt sich der krankheitserhaltende Charakter.

4. Allgemeine Veränderungsresistenz: Es besteht eine Tendenz, einmal als positiv erlebte Situationen erhalten bzw. immer wieder aufsuchen $\mathrm{zu}$ wollen und einmal erfolgreiche Verhaltensmuster immer wieder anzuwenden; und zwar auch dann, wenn sie durch die faktisch fortwährende Veränderung aller überhaupt denkbaren Faktoren objektiv überwiegend negative Aspekte bekommen haben. Am deutlichsten wird dies bei den sogenannten Süchten; in gleichem Maße gilt dies aber etwa auch für die Nichtakzeptierung körperlicher 
Altersveränderungen, für das notwendige Sich-Einstellen auf entstandene Behinderungen, auf krankheitsbedingte Funktionsveränderungen. Im Sinne von krankmachender und krankheitserhaltender Veränderungsresistenz läßt sich auch das Verweilen in Überforderungssituationen verstehen; so hat ja z.B. auch der Streß zwei Seiten, die objektiv gegebene Streßsituation und die subjektive Unfähigkeit, sich ihr zu entziehen (dazu SELIGMANN 1979).

5. Krankheit als Selbstbestrafung: Schuldgefühle, Versündigungsgedanken, Gefühle des eigenen Unwerts lassen Krankheit als etwas Normales, Richtiges, ja Anstrebenswertes erscheinen

6. Krankheit als Selbstaufgabe: Zur Erhaltung und Wiedererlangung seiner Gesundheit braucht der Mensch offenbar das Gefühl, daß diese Gesundheit $\mathrm{zu}$ irgendetwas sie selbst Übersteigende da i st, verwendet wird oder wenigstens werden kann. Es sind akzeptierte und für lösbar gehaltene Aufgaben erforderlich und ein gegenwärtig erkannter Lebenssinn. Heilungsbereitschaft braucht Perspektive; offenbar setzen Gesundbleiben und erst recht Gesundwerden eine Anstrengungsbereitschaft voraus, deren Aufwand durch das schlichte Faktum des Nicht-Krankseins nicht aufgewogen wird. Im Extremfall tritt dadurch der psychogene Tod ein (dazu STUMPFE 1973).

Man kann zusammenfassend sagen, daß es die verschiedenen Funktionen von Krankheit und Gesundsein sind, die die Heilungsbereitschaft beeinflussen und damit die Heilung bei aller gegebenen Heilungsfähigkeit erschweren oder unmöglich machen können. Hilfen, die eine Krankheit und ihre Symptome beseitigen sollen, haben also auch bei den Funktionen dieser Krankheit für den einzelnen und seine Bezugsgruppe und bei der subjektiv empfundenen Bedeutung des Gesundseins anzusetzen. Die von medizinischer Seite erhobene Forderung, nicht die Symptome einer Krankheit zu behandeln, sondern die Krankheit selbst, muß deshalb dahingehend erweitert werden, daß die Krankheit selbst nur ein Symptom sein kann, nämlich für die Probleme und Konflikte, 
die hinter ihr stehen und sie aufrechterhalten.

$\mathrm{Zu}$ dieser Einsicht ist die Psychotherapie und speziell die Psychosomatik gelangt; sie scheint aber auch bei den Heilzauberern wenigstens im Ansatz bereits gegeben zu sein. Im Rahmen der brasilianischen Umbanda wird Krankheit als ein Problem des Patienten unter anderen angesehen oder auch als Hinweis auf tieferliegende Probleme, die allerdings dann natürlich nicht psychische oder psychosoziale, sondern spirituelle genannt werden.

Förderung der Heilungsbereitschaft heißt, die Funktionen der Krankheit überflüssig machen, sie zu kompensieren oder auszuschalten, Gesundheit dagegen auch und gerade in der gegebenen Situation attraktiv erscheinen zu lassen. Dazu gibt es mehrere prinzipiell verschiedene Möglichkeiten. Die eine Gruppe von Möglichkeiten sind die objektiven: Hier geht es um konkrete Veränderungen der Lebensbedingungen des Kranken bzw. darauf hinzielende Beratung. Hilfreich sind je nach Fall ein Berufs- oder Wohnungswechsel, als Luftveränderung kaschiertes Aussteigen aus Alltagsbezügen, Kontakte zu neuen Gruppen, Übernahme neuer konkreter Aufgaben und Verpflichtungen usw. Außerdem können Beratungen zum Ziel führen, die das Selbst- und Weltbild objektivieren, die beispielsweise ins Bewußtsein heben, daß der Kranke krankheitsförderndes Verhalten zeigt, oder daß sein Verhalten erst Reaktionen der Mitmenschen provoziert, an denen er dann leidet, und dergleichen.

Die anderen Möglichkeiten, die Heilungsbereitschaft zu unterstützen, sind nicht objektivierbar. Bei ihnen werden Krankheit und ihre Symptome zugunsten von Konstrukten mehr oder weniger weitgehend ausgeblendet. Es wird auf Umwegen versucht, ohne konkrete Veränderungen der realen Situation und ohne Appell an die Einsicht die Heilungsbereitschaft zu verändern. Dieser Weg wird von den meisten Psychotherapien eingeschlagen und ebenso von Wunderheilern und Zauberpriestern. An die Stelle der Beschäftigung mit realen, objektiven Umständen der Krank- 
heit und der Lebenssituation des Kranken tritt die Beschäftigung mit Konstrukten. Ich bitte, das Wort im wissenschaftlichen Sinn neutral aufzufassen, wenn ich als Konstrukte nebeneinandersetze: Geistereinflüsse, magische Schädigungsversuche, animalischen

Magnetismus, Erdstrahlen, Sünde, libidinöse Strebungen, frühkindliche Traumata, Angstverarbeitungsmechanismen. Dies und vieles andere kann aus der jeweiligen Sicht von Helfenden als Ursache von Krankheiten angesehen oder angegeben werden, wobei es nicht auf die Bewei sbarkei $t$ ankommt, sondern auf die Frage, ob der Kranke solche Erklärungen für sich akzeptiert, d.h. seine Krankheit in entsprechenden Termini zu verstehen bereit ist. Wesentlich ist, daß aus diesen Postulaten heraus Methoden entwickelt worden sind, die tatsächlich die Heilungsbereitschaft und damit die Heilung fördern, sofern der Kranke sie als für sich gültig akzeptiert. Da hier wohl kein prinzipieller Unterschied besteht, kann man alle entsprechenden Verfahren magisch nennen.

Es hat vor Jahrzehnten eine Diskussion darüber gegeben, ob Psychotherapie Magie ist, bzw. wie sie sich von Magie unterscheidet. Da diese Diskussion medizinintern abgelaufen ist, scheint sie keine direkte Auswirkung auf Ethnologie und Ethnomedizin gehabt zu haben. JORES ( o . J . , 1955) hat seinerzeit den Unterschied daran festmachen wollen, daß in der Magie etwas erzwungen werden soll, daß es z. B. auch bei der magischen Wirkung, die von ärztlicher Tätigkeit und ärztlichen Mitteln (z. B. Placebos) ausgeht, um fraglose Unterwerfung geht; in der Psychotherapie dagegen ginge es um Begegnung, um partnerschaftliche Begegnung zwischen Therapeut und Hilfesuchendem. Ich halte eine solche Unterscheidung für ethnozentrisch und inhaltlich unhaltbar. Zum einen geht es auch in der magischen Krankenbehandlung ganz wesentlich um Begegnung mit dem Zauberer, mit den geglaubten übernatürlichen Wesen und Mächten. Daß diese autoritär, besser gesagt: Autoritätsbewußt auftreten, ist eine ganz andere Frage. Auch der 
Psychotherapeut wird keinen Erfolg haben, wenn er unsicher auftritt oder sich dem Patienten gegenüber methodenkritisch äußert. Andererseits muß auch in der Psychotherapie der Hilfesuchende die angebotenen Konstrukte hinnehmen. Wer etwa die symbolische Ausdeutung seiner Träume für Unfug hält, ist darüber ebensowenig therapierbar, wie einer der nicht an die Mani pul ierbarkeit von FI ui den oder ähnliches glaubt.

Magie, die auf die Beseitigung von Leiden und Krankheit zielt, ist Psychotherapie. Umgekehrt sind Psychotherapien, sofern sie nicht auf konkrete Umwelt- und Verhaltensveränderungen über die dazu objektiv geeigneten Mittel gerichtet sind, Magie, die unserer gegenwärtigen Situation entsprechende und deshalb bei uns akzeptierte und wirksame Form von Magie. Doch dies nur zum Stellenwert des traditionellen Heilzaubers.

An dieser Stelle möchte ich eine Bemerkung zu den Ausführungen eines Autors aus ganz anderem wissenschaftlichen Lager einfügen, der noch immer als eine der Autoritäten auf dem Gebiet der Heilmagie zitiert wird: LEVI-STRAUSS. Seine diesbezüglichen Aussagen sind meines Erachtens erstaunlich ethnozentrisch-polemisch und ich fürchte, von ethnomedizinischer Seite ist bisher noch keine klare Distanzierung erfolgt. In "Der Zauberer und seine Magie" (1978: 256-278) bezeichnet LEVI-STRAUSS Zauberer pauschal als "Psychopathen" (1978: 272), die als "professionelle Abreagierer" (1978: 273) dienen. Nach meinen Erfahrungen sind die meisten sogenannten Zauberer normal gesunde, verantwortungsbewußte Menschen; sie sind so viel und so wenig "professionelle Abreagierer" wie unsere eigenen Priester und Therapeuten. Er meint, "der Schamane bietet seinem Publikum ein Schauspiel" (1978: 273); die Gruppe hat damit teil "an jenem Erlebnis einer Welt symbolischer Ergüsse" (1978: 274). Dasselbe könnte ein böswilliger oder nur oberflächlich informierter Heiler beispielsweise von einer katholischen Messe aussagen. Wie unpassend der Ausdruck "Schauspiel" ist, geht schon daraus hervor, daß gleiches oder 
ähnliches Verhalten vom sogenannten Schamanen auch ohne sogenanntes Publikum gezeigt wird; dann nämlich, wenn er mit sich und seinen geglaubten Geistern alleine i st. Und die Wirksamkeit von Geistern durch die Wirksamkeit von Symbolen erklären zu wollen, bedeutet schlicht, ein traditionelles Konstrukt durch ein modernes Konstrukt zu ersetzen.

Am unverständlichsten erscheint mir in genanntem Aufsatz der 1978 zum vielfachen Male veröffentlicht worden ist, die Aussage "die Erfahrungen des Kranken geben den am wenigsten wichtigen Aspekt des Systems wieder" (1978: 272); denn dies scheint mir die Tatsachen geradezu auf den Kopf zu stellen. Natürlich könnte ein Heiler so etwas beispielsweise auch von einem europäischen Krankenhausbetrieb behaupten. Er träfe damit aber wohl kaum den Kern der Sache. Hier wie dort überlebt ein System ja doch wohl nur dann, wenn es wenigstens teilweise das erfüllt, wessentwegen es etabliert worden ist; das heißt im konkreten Fall, wenn tatsächlich Kranken geholfen wird, wenn sie geheilt werden. Und dies ist wenigstens da, wo Heilungsbereitschaft die wesentliche Rolle spielt, nicht anders möglich als über die direkte oder indirekte Veränderung der Erfahrung des Kranken.

Die Mittel, die dazu führen sollen, zu wählen und zu dosieren, ist Sache des Psychotherapeuten, des Heilers, Zauberers, Schamanen. Und er wird sie wählen und dosieren auf dem Hintergrund seiner Persönlichkeit und der Gruppenerwartung, ganz wesentlich und zentral aber im Hinblick auf den Kranken. Wobei dann das sogenannte Publikum zum einen die so wesentliche Bezugsgruppe des Kranken darstellt, zum anderen aber auch mögliche zukünftige Hilfsbedürftige.

\section{Förderung der Heilbereitschaft in der Umbanda}

Im zweiten Teil meiner Ausführungen möchte ich anhand von Beispielen aus der Kultur der brasilianischen Umbanda verdeutlichen, wie die Heilungsbereitschaft beeinflußt werden kann, und insbesondere auch, welche Bedeutung 
dabei dem Heiler und seiner Persönlichkeit zukommt.

Es geht in Umbanda-Veranstaltungen in der Regel ausgesprochen turbulent $\mathrm{zu}$, auch während konzentrierter ernsthafter Beratungsgespräche. Jeder, der in die Kultstätte kommt, wird ganz locker und wie selbstverständlich dazugehörig aufgenommen. Die meist kleinen Räume sind bei solcher Gelegenheit von Menschen überfüllt, es wird getrommmelt und gesungen; Medien in Kultkleidung fallen in Trance und verkörpern Geistrollen, in denen sie mehr oder weniger auffälliges Verhalten zeigen. Auch für die Gläubigen bekommt dies gelegentlich leicht groteske Züge, was jedoch bei der relativen Unberechenbarkeit der geglaubten Geister und ihrem Ernst zur Atmosphäre einer existenziellen Grenzsituation beiträgt.

Aufgrund der Fluidallehre und des Glaubens ans Berufen wird nach Möglichkeit vermieden, Negatives zu erwähnen. Trotz aller möglichen Krankheiten und Leiden, wegen derer Gläubige kommen, wird nie laut davon gesprochen. Auch die Behandlung richtet sich, wie ich noch zeigen werde, nicht eigentlich auf Krankheit und Leiden, sondern auf postulierte und meist realiter neutrale Ursachen. Es herrscht sehr deutlich eine optimistische, zuversichtliche Haltung vor, demonstrierte Lebensbejahung und Lebensbewältigung.

Hinzukommt, daß es im Umbanda-Glauben keinen Zufall gibt. Spätestens in den Gesprächen mit inkorporierten Geistern werden Krankheiten und Leiden in Sinnbezüge gestellt. Damit fühlt sich der Betroffene nicht schlicht ausgeliefert, sondern in höhere Zusammenhänge eingebunden, in denen er und seine Situation eine Funktion haben.

Als günstig erweist sich, daß es immer und unter allen Umständen etwas Sinnvoll-Entscheidendes zu tun gibt, um das eigene Schicksal oder das der Lieben zu beeinflussen. Dabei werden konkrete, mehr oder weniger komplexe und aufwendige Aufgaben gestellt mit Richtung auf transzendente Wesen und Mächte, aber auch in bezug auf die Kultveranstaltung selbst. Diese Aufgaben können sich per se 
niemals als sinnlos erweisen, auch wenn ein erhofftes Ziel durch sie nicht erreicht worden ist.

Aus der Fülle der so erreichbaren psychologischen und psychosomatischen Einflußfaktoren seien genannt: Die Einführung von Zuversicht, damit Auflösung resignativer Inaktivität oder Verkrampfung, die Ablenkung der Aufmerksamkeit von Krankheitssymptomen, Aktivierung von Emotionen und emotionale Umstimmung, die Aufhebung von Vereinsamung oder Auflockerung der Bindung an krankmachende Gruppenstrukturen, die Steigerung des Selbstwertgefühls.

Die Umbanda-Priester sind in ihrer Primärpersönlichkeit meist schwerlich von anderen Leuten $\mathrm{zu}$ unterscheiden. Sie stammen aus verschiedensten sozialen Umgebungen, haben verschiedensten Bildungsstand; viele sind verheiratet, haben Kinder, gehen einem Beruf nach. Sie eignen sich also, wenn man das so sehen will, gut als Identifikationsfiguren. Hervorstechendstes, wohl durchgängiges Merkmal ist ihre überlegene Ruhe und ihr ausgeprägtes, selten zur Schau getragenes Selbstbewußtsein. Außerdem sind sie, sofern man das ohne besondere Untersuchung beurteilen darf, im Durchschnitt psychisch und physisch gesünder als die Normalbevölkerung. Sie entsprechen damit ganz dem, was z. B. LIEK (1931) von $\mathrm{zu}$ seiner Zeit berühmten deutschen Wunderheilern berichtet hat. Interessant ist auch, daß die gesuchtesten Umbands-Priester relativ jung sind. Das entspricht der Feststellung von RUDOLPH (1977: 80), nach der deutsche Heiler von sich aussagen, daß sie mit zunehmendem Alter an 'Kraft' verlieren. Der magische Heiler darf nicht den Eindruck erwecken, daß er sich nicht einmal selbst zu helfen weiß.

Die in der Umbanda sozialisierten Geister bzw. die von Priestern und anderen Aktiven verkörperten Geistrollen sind sehr verschieden; es gibt junge, alte, gute, böse, die alle im Rahmen der Veranstaltungen und der anstehenden Heilungen verschiedene Aufgaben haben. Durchgängig ist aber bei allen 
,folgendes: Sie sind überhaupt nicht beei ndruckbar, zeigen niemals auch nur eine Spur von Unsicherheit oder gar Ängstlichkeit; sie erschrecken unter keinen Umständen; ihre Schmerzempfindlichkeit ist zumeist stark reduziert. Den Gläubigen gegenüber zeigen sie in der Regel kein besonderes Interesse, entsprechend auch eigentlich kein Mitgefühl. Sie halten den Gläubigen auf Distanz, überschreiten des Gläubigen Distanz aber fortwährend, indem sie ihn z.B. völlig frei von Respekt anreden, ihm unverblümt unliebsame Wahrheiten sagen usw. Sie tun so, als wüßten sie im Prinzip alles, selbst wenn sie etwas fragen, und natürlich als könnten sie fast alles. Sie reagieren empfindlich auf Respektlosigkeit oder Herausforderung und gewinnen im Zweifelsfall immer.

Bemerkenswert ist, daß gerade diejenigen Geister besonderen Zulauf haben und als besonders stark geschätzt sind, die relativ viel Angst verbreiten, die Fürchterliches tun und ein ausgesprochen vulgäres Verhalten zeigen. Kommt das Medium dann nach Ausfahren des Geistes wieder zu sich, ist es angepaßt, freundlich und höflich wie vorher. Wahrscheinlich macht gerade dies die Kraft der Überzeugung von übernatürlichen Fähigkeiten aus und damit die Wirksamkeit der Mittel, die zur Mobilisierung der Heilungsfähigkeit führen.

In der Krankenbehandlung der Umbanda interessiert so gut wie nicht, welche Krankheit tatsächlich vorliegt und welche Ursachen, medizinisch gesehen, dahinterstecken. Es geht darum, wie die Krankheit und ihre Ursachen interpretiert werden müssen, damit die zur Verfügung stehenden Mittel angewendet und wirksam werden können. An die Stelle einer Diagnose im medizinischen Sinn tritt deshalb die zweckbestimmte Ursachen-Rekonstruktion. Dabei interessiert, was dem Kranken als Ursache glaubhaft gemacht werden kann, und dies in Zusammenhang damit, was er selbst glaubt, was ihm fehlt und mit was das zusammenhängen mag. UmbandaHeiler arbeiten, wenn man so will, mit Intuition, d. h. sie beobachten scharf (bewußt, meist aber wohl unbewußt) alle Aktionen und Reaktionen des Patienten und formulieren daraus 
ihre eigenen Aussagen. Äußert er z. B. im Zusammenhang mit Klagen über Kopfschmerzen beiläufig den Namen eines ihm unsympathischen Menschen, bietet sich sogleich an, diesen für die Kopfschmerzen verantwortlich zu machen. Wichtiger als der Inhalt von Antworten auf ihre Fragen sind den Geistern offenbar die damit einhergehenden Veränderungen der Körperhaltung, des Tonus, der Transpiration usw. So versteht sich leicht, weshalb manche Geister den Patienten mit geschlossenen Augen bei den Händen halten. Sie erfahren dann auch, auf welche der andeutungsweise vorgeschlagenen Maßnahmen der Patient in der gewünschten Weise, also etwa mit starker emotionaler Beteiligung, reagiert.

Nun möchte ich an einem fiktiven Beispiel die verschiedenen Möglichkeiten durchspielen, so wie ich sie erlebt habe.

1. Rückführung der Krankheit auf negative Fluiden; Behandlung: Passes. Ein Mann mit dauernden Kopfschmerzen wendet sich nach entsprechender allgemeiner Aufforderung durch Gehilfen an einen der inkorporierten Geister, sagen wir Caboclo Weißfeder. Dieser steht leicht schwankend dort, mit blutunterlaufenen Augen, raucht eine schwarze Zigarre, obwohl das Medium vielleicht eine junge Frau ist. Ohne etwas zu fragen, murmelt der Geist mit rauher Stimme einige Begrüßungsworte und beginnt, den Körper des Mannes mit offenen Handflächen zu umfahren. Mit solchen Passes sollen, wie allgemein bekannt ist, negative Fluiden aus dem Körper gezogen werden. Anschließend bläst er Rauch auf den Patienten, was die Übertragung positiver Fluiden andeutet. Nach zwei, drei Minuten ein gemurmelter Segenswunsch; die Behandlung ist abgeschlossen. Obwohl, medizinisch gesprochen, überhaupt nichts passiert ist und weder Fluiden, noch geschweige denn ihre Manipulation, wissenschaftlich gesehen, existieren, haben solche Passes oft nicht nur eine subjektive Wirkung auf den Gläubigen, der sie vielleicht als Entzug von Druck oder als Prickeln der Haut erlebt. Sie können auch eine objektive Wirkung haben insofern, als der 
Glaube an sie beruhigt und entkrampft. Damit können z. B. Kopfschmerzen, die ja häufig nur auf eine Verspannung zurückgehen, schlagartig verschwinden. Dasselbe gilt auch für Krankheiten, die eigentlich nur deshalb weiterbestehen, weil ein subjektiv akzeptabler Auslöser für die Veränderung fehlt. Was gemeint ist, möchte ich an einem alltäglichen Beispiel illustrieren: Wenn man bei jemandem zu Besuch ist, die Zeit vergeht und man sich eigentlich längst verabschieden möchte, tut man es oft erst, nachdem irgendeine zufällige situative Veränderung den Anlaß dafür bietet; wenn also beispielsweise ein Licht an- oder ausgeht oder das Telefon klingelt. Entsprechendes dürfte bei Spontanheilungen der Fall sein; eine psychosomatische Krankheit kann weiterbestehen, obwohl sie ihre ursprüngliche Funktion längst verloren hat und es nur an der Möglichkeit fehlt, sie ohne Gesichtsverlust (auch vor sich selbst) aufzugeben. Hinzukommt dann noch die Funktion, die die Heilung selbst besitzt: Sie bestätigt die Richtigkeit des eigenen Glaubens, des gewählten Heilweges usw.

2. Rückführung der Krankheit auf schwarze Magie; Behandlung: Gegenmagie. Nehmen wir an, der Mann ist durch die Passes seine Kopfschmerzen nicht losgeworden. Er wendet sich an den Priester, der ihm rät an einem anderen Tag wiederzukommen und mit einem anderen Geist zu sprechen, sagen wir, Oma Conga. Am betreffenden Abend fährt Oma Conga in den Körper des Priesters. Die Physiognomie ist verändert, die Stimme nicht wiederzuerkennen. Als der Mann an der Reihe ist, behandelt ihn Oma Conga wie jeden anderen zunächst mit Passes. Plötzlich sieht sie den Mann erstaunt an und sagt: "Mein Sohn, du hast Nadeln im Kopf!" Sie läßt sich bestätigen, daß der Mann unter Kopfschmerzen leidet; läßt sich einiges von seinen Lebensumständen erzählen; sagt, daß sie vor ihrem geistigen Auge eine magische Präparation sieht, die, sagen wir, ein erwähnter Berufskonkurrent gegen ihn durchgeführt hat. "Ich werde dir helfen, mein Sohn. Warte hier, 
ich komme gleich wieder." Der Priester erwacht aus der Trance, blickt sich verwirrt um; der Mann erklärt stammelnd, daß Oma Conga ihm befohlen habe zu warten. Schon fällt der Prieser wieder in Trance, aus seinem Mund lacht Oma Conga: "Habe ich es nicht gesagt, mein Sohn!" Sie ruft einen Gehilfen und läßt aus einem Nebenraum, in dem sich das Allerheiligste befindet, einen Wachskopf holen, in dem Nadeln genau an den Stellen stecken, die der Mann als schmerzend angegeben hat. Oma Conga behauptet, dies sei der apportierte böse Zauber, den sie nun neutralisieren könne. Der Mann erhält irgendwelche Auflagen und im Laufe von mehr oder weniger langer Zeit werden die Nadeln von Oma aus dem Wachskopf gezogen oder mit diesem beseitigt.

Ich habe hier absichtlich das Beispiel einer pia frans gewählt, weil es gleichgültig ist, wie ein magisches Mittel aussieht, wenn nur an seine Wirksamkeit geglaubt wird. Dabei findet zunächst einmal eine intensive Ablenkung des Bewußtseins von der Krankheit bzw. deren Symptomen statt durch nicht ganz durchschaubare interessante, oft auch bedrohlich erscheinende Praktiken. Es kann zu einer Abreaktion gestauter Emotionen kommen, insbesondere, wenn die schwarze Magie angeblich auf den Verursacher zurückgeworfen wird. Auch wird die Einstellung zur Krankheit beeinflußt, es werden unter Umständen bestehende Selbstanklagen, Selbstzweifel, zumindest aber Unsicherheit gemildert. Es kann zu einer Konzentration der Wahrnehmung auf positive Veränderungen kommen, zu einer optimistischen Aktivierung der Körperfunktionen, natürlich negativ auch zu einer Verleugnung der Symptome.

Manche magische Praktiken sind für den Kranken sehr unangenehm und aufwendig, womit der sekundäre Krankheitsgewinn geschmälert werden kann; der Kranke würde dann den Widerstand gegen die Gesundung aufgeben, um sich nicht weiter der Behandlung unterziehen zu müssen.

In bezug auf das Nadel-Beispiel möchte ich noch ein Erlebnis in Ceará erwähnen, bei dem ein Priester eine Hand voll 
Stecknadeln erbrach; Patient war eine junge Ärztin mit der medizinischen Diagnose Krebs. Ob die Behandlung, die natürlich im Rahmen verschiedenster anderer spiritueller Einflußnahmen stattfand, irgendeine Wirkung in bezug auf die Krankheit gehabt hat, kann ich leider nicht sagen; unmöglich ist dies wohl nicht, da inzwischen bekannt ist, daß auch der Krebs psychosoziale Hintergründe hat (LANGENMAYR 1980).

3. Rückführung der Krankheit auf Quälgeister, Krankheitsdämonen; Behandlung: Exorzismus, Opfer. Wenn die magische Prozedur nicht geholfen haben sollte, besteht die Möglichkeit, die Kopfschmerzen auf einen bösen Geist zurückzuführen, der sich im Kranken eingenistet hat oder ihm von außen zusetzt. Der Mann wird von einer Anzahl von Medien umtanzt, während der böse Geist durch Trommeln, Gesänge und Zurufe zum sogenannten Kopfwechsel aufgefordert wird. Uber kurz oder lang stürzt eins der Medien, von Zuckungen befallen zu Boden, schreit, schlägt um sich. Die Verkörperung der Rolle ist außerordentlich beeindruckend, da das Medium sich tatsächlich entweder selbst in Schmerzen am Boden wälzt oder aber als der böse Geist schauerlich lacht, schimpft, extreme Forderungen stellt. Wesentlich ist nun, daß auf diese Weise Wege gesucht und wohl auch nicht selten gefunden werden, auf denen dem Mann geholfen werden kann. Meist handelt es sich um finanziell und zeitlich mehr oder weniger aufwendige Opfer und Gebete, auf die hin der Geist den Kranken in Ruhe lassen soll. Über den Dialog zwischen Gläubigem, Dolmetscher und Geist können aber z.B. schuldhafte Verhaltensweisen zur Sprache kommen, etwa die Nichteinhaltung eines Versprechens gegenüber einem Verstorbenen, oder auch schädliche Neigungen und Verhaltensweisen.

Jedenfalls ist diese Form der Behandlung für den Gläubigen oft erschütternd, wenn nicht unangenehm und peinlich. Neben allem schon Gesagten wirkt hier wohl insbesondere die 
Stiftung extrem negativer Assoziationen im Sinne der Stärkung der Heilungsbereitschaft.

4. Rückführung der Krankheit auf den Wunsch persönlicher Schutzgeister, sich über den Körper des Kranken als Medium äußern zu können; Behandlung: Ausbildung zum Besessenheitsmedium. Ich muß hier zunächst nachtragen, daß die Kranken sich entweder parallel zum Besuch der UmbandaVeranstaltungen in ärztlicher Behandlung befinden oder vom Arzt keine Hilfe bekommen haben; gegebenenfalls werden sie auch sogar von Geistern zum Arztbesuch aufgefordert, dann nämlich, wenn die den Eindruck haben, daß keine spirituellen, wir würden sagen, psychosozialen Ursachen vorliegen. Wenn also eine resistente spirituelle Störung vorzuliegen scheint, ist etwas mit den Schutzgeistern des Menschen nicht in Ordnung. Nach Orakel bef ragung und anderem kann dann dem Kranken empfohlen werden, sich zum Besessenheitsmedium ausbilden zu lassen, um so den eigenen Schutzgeistern die Möglichkeit zu geben, sich regelmäßig im Körper aufzuhalten und sich im Rahmen der Kultveranstaltungen über ihn zu äußern. Dies ist nun eine ganz schwerwiegende, tief in das Leben des Mannes eingreifende Entscheidung. Die Ausbildung ist aus verschiedensten Gründen kostspielig; mit ihr übernimmt er eine Fülle von Verpflichtungen, denen er sich schwer oder überhaupt nicht entziehen kann. Er muß regelmäßig zu Kultveranstaltungen erscheinen, dort soweit ihm das jeweils möglich ist, seine Geistrollen verkörpern, regelmäßig Opfer bringen, Nahrungstabus beachten und anderes. Psychologisch gesprochen stellt die Ausbildung zum Medium einen Eingriff in die psychische Existenz des Menschen dar, bei dem in Trance eine Sekundärpersönlichkeit aufgebaut wird, auf die er von seiner Primärpersönlichkeit und den Alltagsrollen her kaum Einfluß nehmen kann. Die Verantwortung, die hier die Priester besitzen, ist also beträchtlich; sie müssen insbesondere darauf achten, daß die Sekundärpersönlichkeit mit ihren Rollen auf 
das Geschehen in der Kultstätte beschränkt bleibt und an die Auslöser für Erscheinen und Verschwinden gebunden bleibt, die in den Kultveranstaltungen verwendet werden.

Selbstverständlich ist die Sekundärpersönlichkeit, wissenschaftlich gesprochen, ein Teil der Gesamtpsyche des Menschen, so daß die Erfahrungen, die sie macht, auf die Primärpersönlichkeit zurückwirken, auch wenn diese die Erfahrungen nicht selbst macht. Die Verehrung, die dem Geist zuteil wird, die Art, wie auf sein Verhalten reagiert wird, die Erfolge, die er durch Beratung und Behandlung von anderen Gläubigen hat, haben deshalb Einfluß auch auf die Primärpersönlichkeit des betreffenden Menschen. Bemerkenswert ist auch, daß die Medien offenbar die Möglichkeit haben, das eigene Erleben und Verhalten, genauer gesagt, das ihrer Primärpersönlichkeit auf dem Umweg über die Sekundärpersönlichkeit zu beeinflussen. So ist, wenigstens in vielen Kultstätten, eine der ersten Handlungen, die neue Geister erlernen, sich selbst, genauer gesagt, dem eigenen Körper Passes zu geben.

Von zentraler Bedeutung dürfte für die Medien sein, regelmäßig ohne negative Sanktionen einmal Herrscher sein zu dürfen, dann Kind, dann aggressiver Böser, mal Mann, mal Frau; und zwar nicht nur nach den Anforderungen der Situation, sondern auch nach persönlicher Disposition.

Bleibt nachzutragen, daß ich hier die Umbanda-Magie selbstverständlich extrem verkürzt dargestellt habe. Die Priester verfügen nicht nur über ein komplexes Repertoire psychologischer Mittel, mit denen sie in Trance versetzen können, bestimmte Stimmungen erzeugen, Medien wie ihre Geister unter Kontrolle halten und gegebenenfalls auch bestrafen können. Je nach Tradition verwenden sie Kräuter, Kräutersäfte, Mittel, die ehemals auch zu unserem Hausschatz gehörten, wie Wärme- oder Kältebehandlung, Massagen und ähnliches. Im übrigen sind Priester wie Gläubige selbstverständlich in bezug auf das populäre Wissen über Anatomie, Physiologie, Pathologie, Hygiene usw. auf dem 
laufenden. Trotzdem geschieht in der Umbanda zur Heilung von Kranken etwas völlig von der medizinischen Intention, im engeren Sinne des Wortes, Verschiedenes; etwas, was als Steigerung der Heilungsbereitschaft verstanden werden kann. Dabei geht es nicht um die Krankheit, sondern um den Menschen, der sie hat; und es geht beim Versuch der Heilungshilfe nicht um sozusagen technische Manipulationen, sondern um das Angebot von Mitteln im ursprünglichen Sinn des Wortes, mit dem Heiler als Mittler, als Vermittler, als Medium.

\section{Literatur}

BALLY, Gustav 1961: Einführung in die Psychoanalyse Sigmund Freuds.

JORES, Arthur 1955: Magie und Zauber in der modernen Medizin. Deutsche Medizinische Wochenschrift 8: 915-920

JORES, Arthur o.J.: Magie und Wunder in der Medizin. In: BITTER, Wilhelm (Ed) Magie und Wunder in der Heilkunde: $151-158$

LEVI-STRAUSS, Claude: 1978 Der Zauberer und seine Magie. In: PETZOLD, Leander (Ed) Magie und Religion. Beiträge zu einer Theorie der Magie: 256-278

LANGENMAYR, Arnold 1980: Krankheit als psychosoziales Phänomen: 40-48

LIEK, Erwin 1931 (1951): Das Wunder in der Heilkunde.

RUDOLPH, Ebermut 1977 Die geheimnisvollen Ärzte. Von Gesundbetern und Spruchheilern.

SELIGMANN, Martin E.P. 1979 Erlernte Hilflosigkeit.

STUMPFE, Klaus Dieter 1973 Der psychogene Tod. 